\title{
On the variational noncommutative Poisson geometry
}

\author{
A. V. Kiselev
}

December 25, 2011

\begin{abstract}
We outline the notions and concepts of the calculus of variational multivectors within the Poisson formalism over the spaces of infinite jets of mappings from commutative (non)graded smooth manifolds to the factors of noncommutative associative algebras over the equivalence under cyclic permutations of the letters in the associative words. We state the basic properties of the variational Schouten bracket and derive an interesting criterion for (non)commutative differential operators to be Hamiltonian (and thus determine the (non)commutative Poisson structures). We place the noncommutative jet-bundle construction at hand in the context of the quantum string theory.
\end{abstract}

Introduction. In this brief communication we sum up, without giving any detailed proofs, the main notions and facts about the calculus of noncommutative variational multivectors in terms of the Schouten bracket, and we sketch the construction of the odd (homological) evolutionary vector fields, on the infinite jet spaces for maps of a smooth commutative manifold $M^{n}$ to the factor-algebra of a noncommutative associative algebra $\mathcal{A}$ by the relations of the cyclic invariance $a_{1} \times a_{2} \cdot a_{3} \sim a_{1} \cdot a_{2} \times a_{3}$ and $a_{1} \times a_{2} \sim a_{2} \times a_{1}$ (see [1]), where $\times$ is the ordered concatenation of the words $a_{i}$ written in the alphabet of $\mathcal{A}$ and $\cdot$ is the multiplication.

We formulate the basics of the theory over such noncommutative jet bundles; this direction of research was pioneered in [2]. If, at the end of the day, the target algebra $\mathcal{A}$ is proclaimed (graded-)commutative (and if it satisfies the "smoothness" assumptions), we restore the standard, Gel'fand-Dorfman's calculus of variational multivectors [3, 4]. Alternatively, under the shrinking of the source manifold $M^{n}$, which may be our space-time, to a point (or by postulating that the image of $M^{n}$ in $\mathcal{A}$ is a given element whenever the map $M^{n} \rightarrow \mathcal{A}$ is constant), we reproduce the noncommutative symplectic geometry of [1].

This report is an immediate continuation of the review [5] and we follow the notation and conventions thereof, with the only replacement of $\Sigma^{n}$ and $\boldsymbol{q}$ there by $M^{n}$ and $\boldsymbol{a}$ here, respectively. A proper substantiation and the discussion in more detail, with the transcript of this theory as the $\hbar$-linear slice of the scattering equation $(|1\rangle \cdot|2\rangle) \cdot|3\rangle=|1\rangle \cdot(|2\rangle \cdot|3\rangle)$ in the full quantum geometry [6], will be the subject of the subsequent publication.

This paper is structured as follows. We first rephrase the notion of the variational cotangent superbundle (see [4, 5] and references therein) in the noncommutative setup and formulate the definition of the noncommutative Schouten bracket 【, 』 as the odd Poisson bracket. We relate the odd evolutionary vector fields $\boldsymbol{Q}^{\boldsymbol{\xi}}$ to the operations $\llbracket \boldsymbol{\xi}, \cdot \rrbracket$, where $\boldsymbol{\xi}$ is a noncommutative variational multivector. In these terms, we debate the hamletian "presence" or "absence" of the Leibniz rule for the Schouten bracket $\llbracket$, 』. We affirm the shifted-graded skew-symmetry of $\llbracket, \rrbracket$ and directly verify the Jacobi identity, which stems 
from the usual Leibniz rule for the derivations $\boldsymbol{Q}^{\xi}$ acting on the bracket $\llbracket \boldsymbol{\eta}, \boldsymbol{\omega} \rrbracket$. Later on, we focus on the noncommutative variational Poisson bivectors $\boldsymbol{\pi}$ such that $\llbracket \boldsymbol{\pi}, \boldsymbol{\pi} \rrbracket=0$ or, equivalently, $\frac{1}{2}\left(\boldsymbol{Q}^{\boldsymbol{\pi}}\right)^{2}=0$. We derive an interesting criterion for a (non)commutative linear operator $A$ to be Hamiltonian (resp., for the bivector $\boldsymbol{\pi}=\frac{1}{2}\langle\boldsymbol{b}, A(\boldsymbol{b})\rangle$ to satisfy $\llbracket \boldsymbol{\pi}, \boldsymbol{\pi} \rrbracket=0$ ).

1. Noncommutative jets. Let $M^{n}$ be a smooth oriented $\mathbb{R}$-manifold and $\boldsymbol{x} \in M^{n}$ be a point. Let $\mathcal{A}$ be a noncommutative associative algebra of dimension $m$; denote by $\boldsymbol{a}$ a basis in $\mathcal{A}$. Consider the maps $M^{n} \rightarrow \mathcal{A}$ and construct the infinite jet space $J^{\infty}\left(M^{n} \rightarrow \mathcal{A}\right)=$ : $J^{\infty}\left(\pi^{\mathrm{nC}}\right)$, see [5]. Denote by $(a \cdot)$ and $(\cdot a)$ the operators of left- and right-multiplication by a word $a \in \mathcal{A}$ that is always read from left to right. The total derivative w.r.t. $x^{i}$, $1 \leqslant i \leqslant n$, on $J^{\infty}\left(\pi^{\mathrm{nC}}\right)$ is $\overrightarrow{\mathrm{d}} / \mathrm{d} x^{i}=\vec{\partial} / \partial x^{i}+\sum_{|\sigma| \geqslant 0} \boldsymbol{a}_{\sigma+1_{i}} \vec{\partial} / \partial \boldsymbol{a}_{\sigma}$; the evolutionary derivation $\partial_{\varphi}^{(\boldsymbol{a})}=\sum_{|\sigma| \geqslant 0}\left(\overrightarrow{\mathrm{d}}|\sigma| / \mathrm{d} \boldsymbol{x}^{\sigma}\right)(\varphi) \cdot \vec{\partial} / \partial \boldsymbol{a}_{\sigma}$ acts from the left by the Leibniz rule. Denote by $\bar{\Lambda}^{n}\left(\pi^{\mathrm{nC}}\right)$ the $C^{\infty}\left(J^{\infty}\left(\pi^{\mathrm{nC}}\right)\right)$-module of horizontal forms of the highest $\left(n\right.$-th) degree and by $\bar{H}^{n}\left(\pi^{\mathrm{nC}}\right)$ the respective cohomology w.r.t. the horizontal differential $\overline{\mathrm{d}}=\sum_{i=1}^{n} \mathrm{~d} x^{i} \cdot \overrightarrow{\mathrm{d}} / \mathrm{d} x^{i} ;$ the Cartan differential on $J^{\infty}\left(\pi^{\mathrm{nC}}\right)$ is $\mathrm{d}_{\mathcal{C}}=\mathrm{d}_{\mathrm{dR}}-\overline{\mathrm{d}}$. Denote by $\langle$,$\rangle the \bar{\Lambda}^{n}\left(\pi^{\mathrm{nC}}\right)$-valued coupling between the spaces of variational covectors $\boldsymbol{p}$ and evolutionary vectors $\partial_{\varphi}^{(\boldsymbol{a})}$. By default, we pass to the cohomology and, using the integration by parts, normalize the (non)commutative covectors as follows, $\boldsymbol{p}(\boldsymbol{x},[\boldsymbol{a}])=\sum_{j=1}^{m}\langle$ word $\rangle \cdot \mathrm{d}_{\mathcal{C}} a^{j} \cdot\langle$ word $\rangle \cdot \mathrm{d} \boldsymbol{x}$; one then can freely push $\mathrm{d}_{\mathcal{C}} \boldsymbol{a}$ leftor rightmost using the cyclic invariance. (Due to the structure of $\partial_{\varphi}^{(\boldsymbol{a})}$, the value $\langle\boldsymbol{p}, \varphi\rangle$ is well-defined irrespective of the normalization in $\boldsymbol{p}$.)

Let $A: \boldsymbol{p} \rightarrow \varphi$ be a Noether noncommutative linear matrix operator in total derivatives. The adjoint operator $\vec{A}^{\dagger}$ is defined from the equality $\left\langle\boldsymbol{p}_{1}, A\left(\boldsymbol{p}_{2}\right)\right\rangle=\left\langle\boldsymbol{p}_{2}, \vec{A}^{\dagger}\left(\boldsymbol{p}_{1}\right)\right\rangle$, in which we first integrate by parts and then transport the even covector $\boldsymbol{p}_{2}$ around the circle.

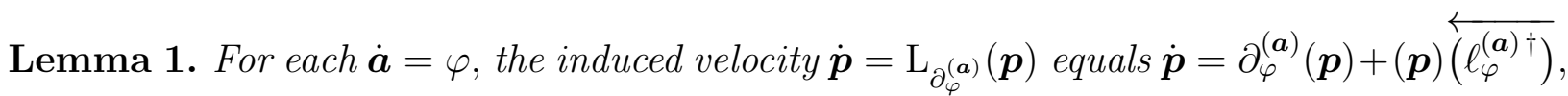
where $\ell_{\varphi}^{(\boldsymbol{a}) \dagger}$ is the adjoint to the linearization, which is $\ell_{\varphi}^{(\boldsymbol{a})}(\boldsymbol{\alpha})=\left.\frac{\mathrm{d}}{\mathrm{d} \varepsilon}\right|_{\varepsilon=0} \varphi(\boldsymbol{x},[\boldsymbol{a}+\varepsilon \boldsymbol{\alpha}])$.

2. Noncommutative multivectors. The covectors $\boldsymbol{p}(\boldsymbol{x},[\boldsymbol{a}])$ were even. We reverse their parity, $\Pi: \boldsymbol{p} \mapsto \boldsymbol{b}(\boldsymbol{x},[\boldsymbol{a}])$, preserving the topology but endowing the space of differential functions $f$ that depend on $\boldsymbol{b}$ with a new ring structure: now, each $f$ is polynomial in finitely many derivatives of $\boldsymbol{b}$. Next, we consider the noncommutative variational cotangent superspace $\overline{J^{\infty}}\left(\Pi \widehat{\pi}_{\pi}^{\mathrm{nC}}\right)=J^{\infty}\left(\Pi \widehat{\pi}^{\mathrm{nC}}\right) \times_{M^{n}} J^{\infty}\left(\pi^{\mathrm{nC}}\right)$, see [3, 4] and [5]. In effect, we declare that $\boldsymbol{b}, \boldsymbol{b}_{\boldsymbol{x}}, \boldsymbol{b}_{\boldsymbol{x} \boldsymbol{x}}, \ldots, \boldsymbol{b}_{\tau}$ are the extra, odd jet variables on top of the old, even $\boldsymbol{a}_{\sigma}$ 's. The total derivatives $\overrightarrow{\mathrm{d}} / \mathrm{d} x^{i}$ obviously lift onto $\overline{J^{\infty}}\left(\Pi \widehat{\pi}_{\pi}^{\mathrm{nC}}\right)$ as well as $\overline{\mathrm{d}}$ that yields the cohomology $\bar{H}^{n}\left(\Pi \widehat{\pi}_{\pi}^{\mathrm{nC}}\right)=\bar{\Lambda}^{n}\left(\Pi \widehat{\pi}_{\pi}^{\mathrm{nC}}\right) /(\mathrm{im} \overline{\mathrm{d}})$. The two components of the evolutionary vector fields $\boldsymbol{Q}=$ $\partial_{\varphi^{a}}^{(\boldsymbol{a})}+\partial_{\varphi^{b}}^{(\boldsymbol{b})}$ now begin with $\dot{\boldsymbol{a}}=\varphi^{\boldsymbol{a}}(\boldsymbol{x},[\boldsymbol{a}],[\boldsymbol{b}])$ and $\dot{\boldsymbol{b}}=\varphi^{\boldsymbol{b}}(\boldsymbol{x},[\boldsymbol{a}],[\boldsymbol{b}])$, c.f. Lemma 1,

The definition of noncommutative variational $k$-vectors, their evaluation on $k$ covectors, the definition of the noncommutative variational antibracket, and its inductive calculation are two pairs of distinct concepts. A noncommutative $k$-vector $\boldsymbol{\xi}, k \geqslant 0$, is a cohomology class in $\bar{H}^{n}\left(\Pi \widehat{\pi}_{\pi}^{\mathrm{nC}}\right)$ whose density is $k$-linear in the odd $\boldsymbol{b}$ 's or their derivatives. Each $\boldsymbol{\xi}$ can be normalized to $\boldsymbol{\xi}=\langle\boldsymbol{b}, A(\boldsymbol{b}, \ldots, \boldsymbol{b})\rangle / k$ !, where the noncommutative total differential operator $A$ depends on $(k-1)$ odd entry and may have $\boldsymbol{a}$-dependent coefficients. Integrating by parts and pushing the letters of the word $\boldsymbol{\xi}$ along the circle, we infer that $\left.\left\langle\boldsymbol{b}_{1}, A\left(\boldsymbol{b}_{2}, \ldots, \boldsymbol{b}_{k}\right)\right\rangle\right|_{\boldsymbol{b}_{i}:=\boldsymbol{b}}=$ $\left.\left\langle\boldsymbol{b}_{2}, A_{\circlearrowright}^{\dagger}\left(\boldsymbol{b}_{3}, \ldots, \boldsymbol{b}_{k}, \boldsymbol{b}_{1}\right)\right\rangle\right|_{\boldsymbol{b}_{i}:=\boldsymbol{b}} ;$ note that, each time an odd variable $\boldsymbol{b}_{\tau}$ reaches a marked point $\infty$ on the circle, it counts the $k-1$ other odd variables whom it overtakes and reports the sign $(-)^{k-1}$ (in particular, $A_{\circlearrowright}^{\dagger}=A^{\dagger}=-A$ if $k=2$ ). The value of the $k$-vector $\boldsymbol{\xi}$ on $k$ arbitrary 
covectors $\boldsymbol{p}_{i}$ is $\boldsymbol{\xi}\left(\boldsymbol{p}_{1}, \ldots, \boldsymbol{p}_{k}\right)=\sum_{s \in S_{k}}(-)^{|s|}\left\langle\boldsymbol{p}_{s(1)}, A\left(\boldsymbol{p}_{s(2)}, \ldots, \boldsymbol{p}_{s(k)}\right)\right\rangle / k !$; we emphasize that we shuffle the arguments but never swap their slots, which are built into the cyclic word $\boldsymbol{\xi}$.

3. Noncommutative Schouten bracket. The concatenation $\times$ of densities of two multivectors provides an ill-defined product in $\bar{H}^{n}\left(\Pi \widehat{\pi}_{\pi}^{\mathrm{nC}}\right)$, where the genuine multiplication is the noncommutative antibracket. We fix the Dirac ordering $\delta \boldsymbol{a} \wedge \delta \boldsymbol{b}$ over each $\boldsymbol{x}$ in $\overline{J \infty}\left(\Pi \widehat{\pi}_{\pi}^{\mathrm{nC}}\right) \rightarrow M^{n}$; note that $\delta \boldsymbol{a}$ is a covector and $\delta \boldsymbol{b}$ is an odd vector so that their coupling equals $+1 \cdot \mathrm{d} \boldsymbol{x}$. The noncommutative variational Schouten bracket of two multivectors $\boldsymbol{\xi}$ and $\boldsymbol{\eta}$ is $\llbracket \boldsymbol{\xi}, \boldsymbol{\eta} \rrbracket=\langle\vec{\delta} \boldsymbol{\xi} \wedge \overleftarrow{\delta} \boldsymbol{\eta}\rangle$. In coordinates, this yields $\llbracket \boldsymbol{\xi}, \boldsymbol{\eta} \rrbracket=[\vec{\delta} \boldsymbol{\xi} / \delta \boldsymbol{a} \cdot \overleftarrow{\delta} \boldsymbol{\eta} / \delta \boldsymbol{b}-\vec{\delta} \boldsymbol{\xi} / \delta \boldsymbol{b} \cdot \overleftarrow{\delta} \boldsymbol{\eta} / \delta \boldsymbol{a}]$, where (1) all the derivatives are thrown off the variations $\delta \boldsymbol{a}$ and $\delta \boldsymbol{b}$ via the integration by parts, then (2) the letters $\boldsymbol{a}_{\sigma}, \boldsymbol{b}_{\tau}, \delta \boldsymbol{a}$, and $\delta \boldsymbol{b}$, which are thread on the two circles $\delta \boldsymbol{\xi}$ and $\delta \boldsymbol{\eta}$, spin along these rosaries so that the variations $\delta \boldsymbol{a}$ and $\delta \boldsymbol{b}$ match in all possible combinations, and finally, (3) the variations $\delta \boldsymbol{a}$ and $\delta \boldsymbol{b}$ detach from the circles and couple, while the loose ends of the two remaining open strings join and form the new circle.

The Schouten bracket is shifted-graded skew-symmetric: if $\boldsymbol{\xi}$ is a $k$-vector and $\boldsymbol{\eta}$ an $\ell$-vector, then $\llbracket \boldsymbol{\xi}, \boldsymbol{\eta} \rrbracket=-(-)^{(k-1)(\ell-1)} \llbracket \boldsymbol{\eta}, \boldsymbol{\xi} \rrbracket$.

4. Is $\llbracket, \rrbracket$ a bi-derivation? In the notation of $\S 3$, define the evolutionary vector field $Q^{\xi}$ on $\overline{J^{\infty}}\left(\Pi \widehat{\pi}_{\pi}^{\mathrm{nC}}\right)$ by the rule $\boldsymbol{Q}^{\boldsymbol{\xi}}(\boldsymbol{\eta})=\llbracket \boldsymbol{\xi}, \boldsymbol{\eta} \rrbracket$, whence $\boldsymbol{Q}^{\boldsymbol{\xi}}=-\partial_{\vec{\delta} \boldsymbol{\xi} / \delta \boldsymbol{b}}^{(\boldsymbol{a})}+\partial_{\vec{\delta} \boldsymbol{\xi} / \delta \boldsymbol{a}}^{(\boldsymbol{b})}$. The normalization $\boldsymbol{\xi}=\left\langle\boldsymbol{b}, A(\boldsymbol{b}, \ldots, \boldsymbol{b}\rangle / k\right.$ ! determines $\boldsymbol{Q}^{\boldsymbol{\xi}}=-(-)^{k-1} \frac{1}{(k-1) !} \partial_{A(\boldsymbol{b}, \ldots, \boldsymbol{b})}^{(\boldsymbol{a})}+\left.(-)^{k-1} \frac{1}{k !} \partial_{\vec{\ell}_{A\left(\boldsymbol{b}_{2}, \ldots, b_{k}\right)}^{(\boldsymbol{a}) \dagger}\left(\boldsymbol{b}_{1}\right)}^{\left(\boldsymbol{a}_{1}\right)}\right|_{\boldsymbol{b}_{i}:=\boldsymbol{b}} ;$ e.g., $\boldsymbol{Q}^{\frac{1}{2}\langle\boldsymbol{b}, A(\boldsymbol{b})\rangle}=\partial_{A(\boldsymbol{b})}^{(\boldsymbol{a})}-\frac{1}{2} \partial_{\vec{\ell}_{A(\boldsymbol{b})}^{(\boldsymbol{a}) \dagger}(\boldsymbol{b})}^{(\boldsymbol{b})}$, see [4, [5].

Freeze the coordinates, fix the volume form on $M^{n}$, and choose any representatives $\boldsymbol{\xi}$ and $\boldsymbol{\eta}$ of the cohomology classes in $\bar{H}^{n}\left(\Pi \widehat{\pi}_{\pi}^{\mathrm{nC}}\right)$. The derivation $\boldsymbol{Q}^{\xi}$ acts on the word $\boldsymbol{\eta}$ by the graded Leibniz rule, inserting $\frac{\overrightarrow{\mathrm{d}}^{|\sigma|}}{\mathrm{d} \boldsymbol{x}^{\sigma}}\left(\boldsymbol{Q}^{\xi}(\boldsymbol{q})\right.$ ) instead of each letter $\boldsymbol{q}_{\sigma}$ (here $\boldsymbol{q}$ is $\boldsymbol{a}$ or $\boldsymbol{b}$ ). Next, promote the letter $\boldsymbol{q}$ to the zero- or one-vector $\boldsymbol{q} \cdot \mathrm{d} \boldsymbol{x} \in \bar{\Lambda}^{n}\left(\Pi \widehat{\pi}_{\pi}^{\mathrm{nC}}\right)$ and use the Leibniz rule again to expand the entries $\llbracket \boldsymbol{\xi}, \boldsymbol{q} \rrbracket=(\boldsymbol{\xi}) \overleftarrow{\boldsymbol{Q}^{q}}$. The bracket $\llbracket, \rrbracket: \bar{\Lambda}^{n}\left(\Pi \widehat{\pi}_{\pi}^{\mathrm{nC}}\right) \times \bar{\Lambda}^{n}\left(\Pi \widehat{\pi}_{\pi}^{\mathrm{nC}}\right) \rightarrow$ $\bar{\Lambda}^{n}\left(\Pi \widehat{\pi}_{\pi}^{\mathrm{nC}}\right)$ becomes a derivation in each argument. However, the calculation of $\llbracket \boldsymbol{\xi}, \boldsymbol{\eta} \rrbracket$ is ill-defined if one permits the addition of $\bar{d}$-exact terms (e.g., stemming from the integration by parts) to the entries $\llbracket \boldsymbol{\xi}, \boldsymbol{q} \rrbracket$. Besides, the normalization of the final result is a must in order to let us compare any given multivectors; in fact, the usual commutator of one-vectors is always transformed to $\left\langle\boldsymbol{b},-\left(\partial_{\varphi_{1}}^{(\boldsymbol{a})}\left(\varphi_{2}\right)-\partial_{\varphi_{2}}^{(\boldsymbol{a})}\left(\varphi_{1}\right)\right)\right\rangle=\llbracket\left\langle\boldsymbol{b}, \varphi_{1}\right\rangle,\left\langle\boldsymbol{b}, \varphi_{2}\right\rangle \rrbracket$, with no derivatives falling on $\boldsymbol{b}$. At this point, the Leibniz rule for $\llbracket$, $\rrbracket$ is in general irreparably lost.

The conceptual equality $\left[\boldsymbol{Q}^{\xi}, \boldsymbol{Q}^{\eta}\right]=\boldsymbol{Q}^{\llbracket \xi, \eta \rrbracket}$, with the graded commutator in its 1.-h.s. and the noncommutative variational Schouten bracket in the r.-h.s., proves that the Leibniz rule $\boldsymbol{Q}^{\boldsymbol{\xi}}(\llbracket \boldsymbol{\eta}, \boldsymbol{\omega} \rrbracket)=\llbracket \boldsymbol{Q}^{\boldsymbol{\xi}}(\boldsymbol{\eta}), \boldsymbol{\omega} \rrbracket+(-)^{(k-1)(\ell-1)} \llbracket \boldsymbol{\eta}, \boldsymbol{Q}^{\boldsymbol{\xi}}(\boldsymbol{\omega}) \rrbracket$, where $\boldsymbol{\omega} \in \bar{H}^{n}\left(\Pi \widehat{\pi}_{\pi}^{\mathrm{nC}}\right)$, is the Jacobi identity $\llbracket \boldsymbol{\xi}, \llbracket \boldsymbol{\eta}, \boldsymbol{\omega} \rrbracket \rrbracket=\llbracket \llbracket \boldsymbol{\xi}, \boldsymbol{\eta} \rrbracket, \boldsymbol{\omega} \rrbracket+(-)^{(k-1)(\ell-1)} \llbracket \boldsymbol{\eta}, \llbracket \boldsymbol{\xi}, \boldsymbol{\omega} \rrbracket \rrbracket$ for the bracket $\llbracket, ~ \rrbracket$.

5. Noncommutative Poisson brackets. Each skew-adjoint noncommutative linear total differential operator $A: \boldsymbol{p} \mapsto \dot{\boldsymbol{a}}=\varphi$ yields the bivector $\boldsymbol{\pi}=\frac{1}{2}\langle\boldsymbol{b}, A(\boldsymbol{b})\rangle$. Let $\mathcal{H}_{1}, \mathcal{H}_{2}, \mathcal{H}_{3}$ be zero-vectors, i.e., $\mathcal{H}_{i}=\left[h_{i}(\boldsymbol{x},[\boldsymbol{a}]) \mathrm{d} \boldsymbol{x}\right]$. By definition, put $\left\{\mathcal{H}_{i}, \mathcal{H}_{j}\right\}_{A}:=\boldsymbol{\pi}\left(\vec{\delta} \mathcal{H}_{i} / \delta \boldsymbol{a}, \vec{\delta} \mathcal{H}_{j} / \delta \boldsymbol{a}\right)$, which equals $\left\langle\vec{\delta} \mathcal{H}_{i} / \delta \boldsymbol{a}, A\left(\vec{\delta} \mathcal{H}_{j} / \delta \boldsymbol{a}\right)\right\rangle=\partial_{A\left(\vec{\delta} \mathcal{H}_{j} / \delta \boldsymbol{a}\right)}^{(\boldsymbol{a})}\left(\mathcal{H}_{i}\right)(\bmod \mathrm{im} \overline{\mathrm{d}})$. The bracket $\{,\}_{A}$ is bilinear and skew-symmetric (but it does not restrict as a bi-derivation to the cohomology w.r.t. $\overline{\mathrm{d}}$ ); it becomes Poisson if it satisfies the Jacobi identity $\sum_{\mathcal{O}}\left\{\left\{\mathcal{H}_{1}, \mathcal{H}_{2}\right\}_{A}, \mathcal{H}_{3}\right\}_{A}=0$, which also is $\sum_{s \in S_{3}}(-)^{|s|} \partial_{A\left(\vec{\delta} \mathcal{H}_{s(3)} / \delta \boldsymbol{a}\right)}^{(\boldsymbol{a})}\left(\frac{1}{2}\left\langle\vec{\delta} \mathcal{H}_{s(1)} / \delta \boldsymbol{a}, A\left(\vec{\delta} \mathcal{H}_{s(2)} / \delta \boldsymbol{a}\right)\right\rangle\right)=0$. The tempting no- 
tation $\partial_{A(\boldsymbol{b})}^{(\boldsymbol{a})}(\boldsymbol{\pi})\left(\bigotimes^{3} \vec{\delta} \mathcal{H}_{i} / \delta \boldsymbol{a}\right)=0$ is illegal by Lemma 1 that forbids us to set $\dot{\boldsymbol{p}} \equiv 0$ at will so that $\partial_{A(\boldsymbol{b})}^{(\boldsymbol{a})}$ would be ill-defined on $\overline{J^{\infty}}\left(\Pi \widehat{\pi}_{\pi}^{\mathrm{nC}}\right)$. Instead, we step farther and reach the classical noncommutative master equation $\boldsymbol{Q}^{\boldsymbol{\pi}}(\boldsymbol{\pi})=\llbracket \boldsymbol{\pi}, \boldsymbol{\pi} \rrbracket=0$ upon the Poisson structures.

Criterion (see [5, 7] on $\frac{1}{2}\left(\boldsymbol{Q}^{\boldsymbol{\pi}}\right)^{2}=0$ ). A skew-adjoint (non) commutative linear matrix operator $A: \boldsymbol{p} \mapsto \dot{\boldsymbol{a}}=\varphi$ in total derivatives is Hamiltonian-i.e., the bivector $\boldsymbol{\pi}$ is Poisson-if and only if its image is involutive: $[\operatorname{im} A, \operatorname{im} A] \subseteq \operatorname{im} A$.

Remark. The construction of $\llbracket, \rrbracket$ in $\S 3$ is the standard string theory's pair of pants $\Sigma_{|i\rangle}$. $\Sigma_{|j\rangle} \mapsto \Sigma_{|i \cdot j\rangle}$ flying over the Minkowski space-time $M^{3,1}$; the dimension reduction is not required. Neither the diameters of the circles $\Sigma \simeq \mathbb{S}^{1}$ that carry the words nor their stretching or oscillations on them, but it is the information about the cyclic order that matters.

Conclusion. The linking of the words into circles is natural for string theory, so the calculus of noncommutative variational multivectors may give new insights in it. Yet, for the alphabet of $\mathcal{A}$ to fully depict the quantum world, we must quantize both the algebra and the Poisson brackets $\{,\}_{A}$ to their $\hbar$-deformations $\mathcal{A}[\hbar]$ and $\{,\}_{A[\hbar]}^{[\hbar]}$. This will be discussed elsewhere.

Acknowledgements. The author is grateful to the Organizing committee of the international workshop SQS'11 'Supersymmetry and Quantum Symmetries' (July 18-23, 2011; JINR Dubna, Russia) for a welcome and warm atmosphere during the meeting. The author thanks B. A. Dubrovin, E. A. Ivanov, S. O. Krivonos, J. W. van de Leur, P. J. Olver, and V. V. Sokolov for helpful discussions and stimulating remarks.

This research was supported in part by NWO VENI grant 639.031.623 (Utrecht) and JBI RUG project 103511 (Groningen). A part of this research was done while the author was visiting at the IHÉS (Bures-sur-Yvette); the financial support and hospitality of this institution are gratefully acknowledged.

\section{References}

[1] Kontsevich M. Formal (non)commutative symplectic geometry // The Gel'fand Mathematical Seminars, 1990-1992. Boston, MA: Birkhäuser, 1993. P. 173-187.

[2] Olver P. J., Sokolov V. V. Integrable evolution equations on associative algebras // Commun. Math. Phys. 1998. V. 193, n. 2. P. 245-268.

[3] Olver P. J. Applications of Lie groups to differential equations. Grad. Texts in Math. V. 107 (2nd ed.). N.Y.: Springer-Verlag, 1993.

[4] Krasil'shchik I., Verbovetsky A. Geometry of jet spaces and integrable systems // J. Geom. Phys. 2011. V. 61. P. 1633-1674.

[5] Kiselev A. V. Homological evolutionary vector fields in Korteweg-de Vries, Liouville, Maxwell, and several other models. Preprint IHÉS/M/11/26. 2011. 20 p.

[6] Kontsevich M. Deformation quantization of Poisson manifolds. I // Lett. Math. Phys. 2003. V. 66, n. 3. P. 157-216. arXiv:q-alg/9709040

[7] Kiselev A. V., van de Leur J. W. Variational Lie algebroids and homological evolutionary vector fields // Theor. Math. Phys. 2011. V. 167, n.3. P. 772-784. 